\title{
Non-attended data acquisition in the protein lab using the SCOUT sample changer
}

\author{
Michael Mrosek \\ Bruker AXS GmbH, Karlsruhe, Germany; \\ michael.mrosek@bruker.com
}

Unattended X-ray data collection of macromolecules is in increasing demand by an ever more diverse research community, both academic and industrial, especially under the current situation of restricted access to research facilities due to the global pandemic. To better serve the user's needs, and to allow automated and high-throughput operation, a sample changer that can perform autonomous crystal screening and data collection of up to 48 samples per session has been developed. The SCOUT sample changer centers the sample initially by means of visual loop centering in conjunction with feature recognition algorithms. In the case of opaque samples, centering by means of orthogonal X-ray scans can also be performed. The samples are kept safe in a custom designed, twin dewar system to minimize ice buildup upon storage and operation, while the six-axis, collaborative robotic arm is mounted on the enclosure ceiling to ensure a minimal footprint during manual operation. The system can be fitted to any D8VENTURE platform providing an exciting upgrade path to existing laboratory hardware. A comprehensive software package completes the system providing fully customizable, automatic routines for crystal screening, strategy determination, data collection and further downstream data processing.

Keywords: Sample Changer SCOUT non-attended protein lab X-ray centering 